\title{
Abnormal Processing of Social Information from Faces in Autism
}

\author{
Ralph Adolphs, Lonnie Sears, and Joseph Piven
}

\begin{abstract}
Autism has been thought to be characterized, in part, by dysfunction in emotional and social cognition, but the pathology of the underlying processes and their neural substrates remain poorly understood. Several studies have hypothesized that abnormal amygdala function may account for some of the impairments seen in autism, specifically, impaired recognition of socially relevant information from faces. We explored this issue in eight high-functioning subjects with autism in four experiments that assessed recognition of emotional and social information, primarily from faces. All tasks used were identical to those previously used in studies of
\end{abstract}

\section{INTRODUCTION}

Autism is a developmental neuropsychiatric syndrome defined by deficits in social behavior and communication, and by stereotyped, repetitive behaviors, which show a characteristic course. Although most autistic subjects show impairments in multiple cognitive domains, there is some evidence that high-functioning autism may be a disorder that disproportionately affects those aspects of cognition most relevant to social functioning. The theoretical support for this idea comes from the hypothesis that social cognition may be functionally modular, in the sense that it can be, in principle, dissociated from other aspects of cognition, and in the sense that relatively dedicated neural systems may have evolved to subserve some of its component processes (Karmiloff-Smith, Bellugi, Klima, Grant, \& Baron-Cohen, 1995; see also for a review Adolphs, 1999). The empirical support for this idea comes from findings that suggest subjects with autism can be impaired relatively selectively on tasks that assess their knowledge of other people's mental states, with relative sparing of other perceptual and cognitive abilities (see for a review Baron-Cohen, 1995).

In regard to processing social information, high-functioning subjects with autism (we use the term "highfunctioning autism" interchangeably with "Asperger Syndrome") show relatively selective impairments in

University of Iowa subjects with bilateral amygdala damage, permitting direct comparisons. All subjects with autism made abnormal social judgments regarding the trustworthiness of faces; however, all were able to make normal social judgments from lexical stimuli, and all had a normal ability to perceptually discriminate the stimuli. Overall, these data from subjects with autism show some parallels to those from neurological subjects with focal amygdala damage. We suggest that amygdala dysfunction in autism might contribute to an impaired ability to link visual perception of socially relevant stimuli with retrieval of social knowledge and with elicitation of social behavior. recognizing higher-order mental states from faces. Baron-Cohen, Wheelwright, and Jolliffe (1997) found that higher-order social/mental states, which were signalled primarily by the eyes (e.g., states such as "flirtatiousness"), were not recognized normally by subjects with high-functioning autism. However, the subjects in that study were readily able to recognize the facial expression of other basic emotions such as happiness (cf. also Baron-Cohen, Spitz, \& Cross, 1993). These findings from tasks involving recognition of social and emotional information from faces show some parallels to a variety of other tasks that have been used to assess the processes by which we normally attribute mental states to other individuals. In particular, Baron-Cohen (1995) and others (Leslie, 1987) have argued that the social impairments seen in autism result in large part from an impaired ability to use a "theory of mind" mechanism. The details of this latter hypothesis are contentious, and in the present paper, we do not wish to take any particular stance on that issue.

The neurobiological basis for autism is a topic of intense recent research investigations (Piven, 1997). It remains unclear to what extent the abnormal processing of social and emotional information in subjects with autism could be due, at least in part, to dysfunction in specific limbic neural structures, such as the amygdala. The proposal that amygdala pathology could contribute to some of the neuropsychological impairments in social and emotional processing seen in autism (Baron-Cohen et al., 2000) is supported by the finding that subjects 
with damage to the amygdala also show abnormal emotional and social processing. In particular, several studies using either subjects with amygdala lesions (Broks et al., 1998; Adolphs, Tranel, Damasio, \& Damasio, 1994; Adolphs et al., 1999), or using functional imaging of the amygdala in normal individuals (Breiter et al., 1996; Morris et al., 1996), have demonstrated that the amygdala is important for recognition of certain emotions, such as fear, from facial expressions, and that it is also important for making more complex social judgments about faces, such as their perceived trustworthiness (Adolphs, Tranel, \& Damasio, 1998). Further support for a possible link between amygdala dysfunction and autism is provided by a recent functional imaging study, which showed that the amygdala is activated in normal individuals, but not in subjects with autism, on a task in which autistic subjects are impaired (Baron-Cohen et al., 1999; see also Baron-Cohen et al., 2000).

In addition to these investigations of the functions of the human amygdala, Bauman and Kemper (1985) found morphological abnormalities in the amygdalas of autistic subjects, and structural MRI studies have also reported differences between amygdala volume in highfunctioning autistic subjects compared to normal controls (Abell et al., 1999). A direct functional role for the amygdala has been proposed from animal studies: Experimental lesions of the amygdala in young monkeys (Bachevalier, 1991) result in social impairments that bear some resemblance to the abnormal behavior seen in human autism. All these disparate findings suggest the hypothesis that impaired emotional and social cognition in autism may be, in part, caused by amygdala dysfunction (Baron-Cohen et al., 2000; Damasio \& Maurer, 1978). However, to date, no investigations have specifically compared subjects with amygdala lesions to subjects with autism on the same tasks.
The present study provides such a direct comparison. We used four experimental tasks to assess the recognition of emotional and social information from faces, as well as from lexical stimuli, in eight high-functioning subjects diagnosed with autism. All of the tasks were identical in both format and in stimuli to tasks previously published on subjects with amygdala lesions. Data from subjects with autism were compared to those from normal controls and from subjects with bilateral amygdala damage. All data from normal subjects and from subjects with bilateral amygdala damage have been published previously in several reports (Adolphs et al., 1994; Adolphs, Tranel, Damasio, \& Damasio, 1995; Adolphs et al., 1998, 1999). We here provide summaries of the findings from these prior studies for the purpose of direct comparisons with autistic subjects.

Due to time and testing constraints, not all of the eight subjects participated on all tasks; details on all subjects are provided in Tables 1 and 2. Taken together, the data permit a detailed investigation of how emotional and social stimuli are processed by subjects with autism, and further allow direct comparisons with the performances on the same tasks given by neurological patients with focal brain damage, with a special emphasis on the amygdala.

\section{RESULTS}

\section{Experiment 1: Discriminating the Intensity of Facial Emotion (Jansari, Tranel, \& Adolphs, 2000; Adolphs et al., 1998)}

Subjects with autism did not have any visuoperceptual impairments in processing faces. Their abilities to discriminate faces on the basis of identity were in the normal range on a standard neuropsychological test (the Benton Facial Recognition Task; Benton, Hamsher, Varney, \& Spreen, 1983; Table 1). Similarly, their ability

Table 1. Demographic and Background Neuropsychological Information on Participants with Autism

\begin{tabular}{|c|c|c|c|c|c|c|c|}
\hline \multirow[b]{2}{*}{ ID No. } & \multirow[b]{2}{*}{ Age } & \multirow[b]{2}{*}{$P I Q$} & \multirow[b]{2}{*}{ VIQ } & \multirow[b]{2}{*}{ Benton } & \multicolumn{3}{|c|}{ Autism Diagnostic Interview } \\
\hline & & & & & Social & Communication & Repetitive \\
\hline 1 & 21 & 130 & 108 & 71 & 18 & 8 & 5 \\
\hline 2 & 17 & 120 & 90 & 71 & 27 & 21 & 8 \\
\hline 4 & 28 & 115 & 90 & 90 & 38 & 24 & 9 \\
\hline 5 & 24 & 117 & 88 & 90 & 25 & 13 & 4 \\
\hline 6 & 21 & 109 & 127 & 71 & 24 & 12 & 7 \\
\hline 7 & 21 & 91 & 119 & 22 & 21 & 13 & 9 \\
\hline 8 & 19 & 93 & 84 & 85 & 15 & 15 & 5 \\
\hline 10 & 18 & 91 & 96 & 22 & 12 & 14 & 4 \\
\hline
\end{tabular}

Subscale scores are shown for the Autism Diagnostic Interview (LeCouteur et al., 1989); percentiles for the Benton Facial Recognition Task (Benton et al., 1983), a measure of the ability to discriminate face identity. IQ scores are from the WAIS-R or WISC-R. 
Table 2. Summary of Sample Sizes, Ages, and IQs for the Different Subject Groups and Experiments

\begin{tabular}{lllll}
\hline & & Autistic & Normal & Amygdala \\
\hline$N$ & Experiment 1 & 6 & 28 & 3 \\
& Experiment 2 & 7 & 18 & 8 \\
& Experiment 3 & 8 & 47 & 3 \\
& Experiment 4 & 5 & 20 & 3 \\
Age & Experiment 1 & $22 \pm 3$ & $43 \pm 16$ & $47 \pm 18$ \\
& Experiment 2 & $21 \pm 4$ & $56 \pm 16$ & $53 \pm 17$ \\
& Experiment 3 & $21 \pm 4$ & $19 \pm 1$ & $47 \pm 18$ \\
& Experiment 4 & $23 \pm 3$ & $19 \pm 1$ & $47 \pm 18$ \\
IQ & Experiment 1 & $106 \pm 16$ & - & $98 \pm 9$ \\
& Experiment 2 & $102 \pm 15$ & - & - \\
& Experiment 3 & $104 \pm 15$ & - & $98 \pm 9$ \\
& Experiment 4 & $109 \pm 15$ & - & $98 \pm 9$ \\
\hline
\end{tabular}

to discriminate faces on the basis of the intensity of emotional expression (Experiment 1; Figure 1) did not differ from normal controls. No autistic subject was more than two $S D$ below the normal mean in the accuracy with which they discriminated very faint morphs of emotional facial expressions from neutral faces. These findings are particularly relevant, since they demonstrate normal discrimination for the same

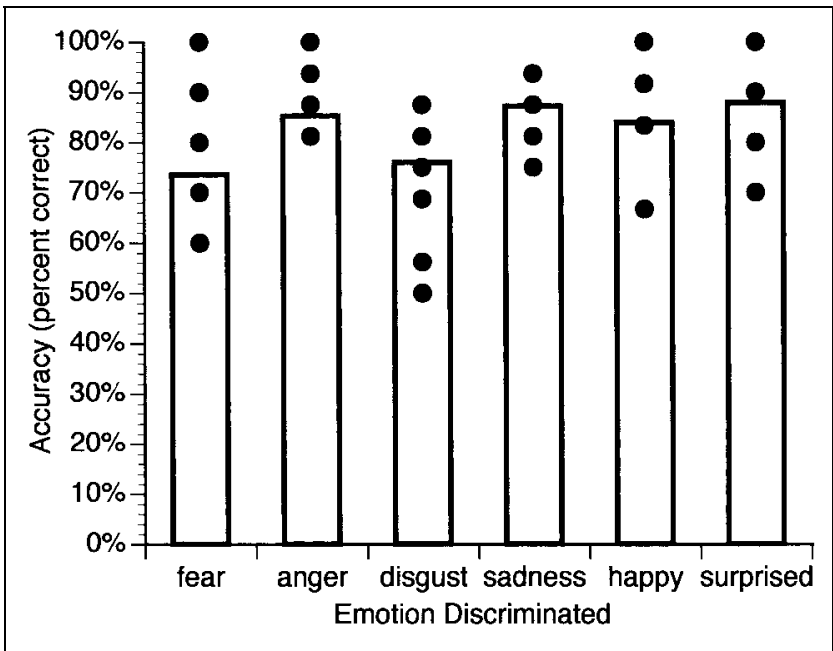

Figure 1. Discrimination of emotional facial expressions. Accuracy in discriminating morphs of the six basic emotions in facial expressions is shown as \% correct on a two-alternative forced-choice task $(50 \%$ is chance), for six autistic subjects (filled circles) compared to the mean of 28 normal controls (bars). Every autistic subject performed within two $S D$ of the control mean. class of stimuli that we used in further tasks reported below.

\section{Experiment 2. Recognition of Basic Emotions from Facial Expressions (Adolphs et al., 1994, 1995, 1999)}

Most autistic subjects tested gave normal ratings to facial expressions of happiness, surprise, anger, disgust, sadness, and fear (Figure 2). Their performances on this task were in general within the range given by normal subjects (Figure 2, gray bars). As a group, the performance of subjects with autism $(n=7)$ was better on all emotions than were the performances of subjects with bilateral amygdala damage $(n=8)$. A repeated-measures ANOVA with a within-subjects factor of emotion type (happy, surprised, afraid, angry, disgusted, or sad) and a between-subjects factor of subject group (autistic, amygdala damage, normal control) revealed significant effects of both emotion $[F(5)=18 ; p<.0001]$ and subject group $[F(2)=5.1 ; p<.01]$, but no interaction between the two.

There were some provocative individual performances. Notably, one of the autistic subjects (Subject

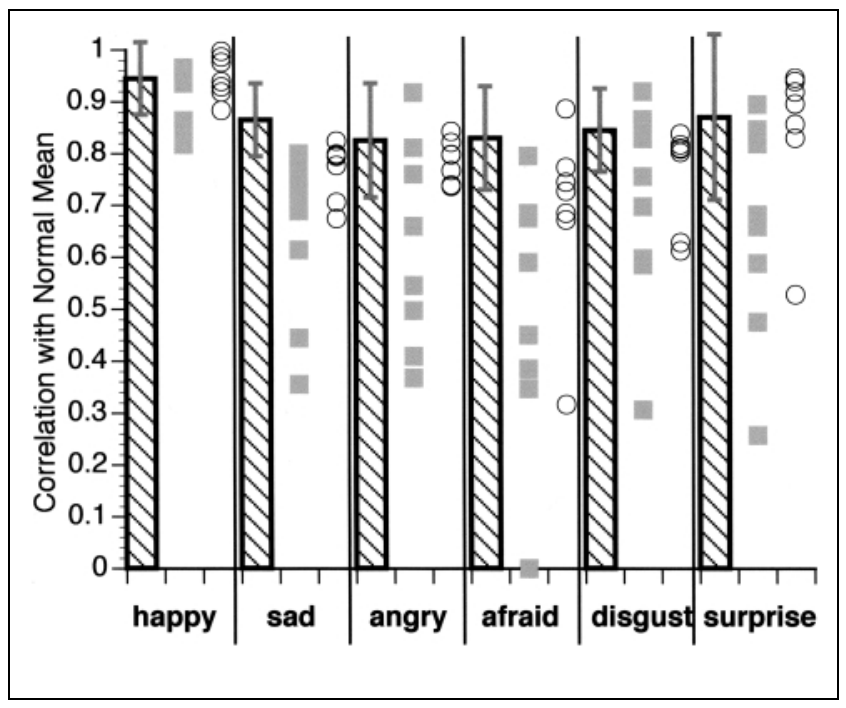

Figure 2. Recognition of basic emotions in facial expressions. Shown are correlations between ratings given by autistic subjects with mean normal ratings (circles), compared to the same correlation measures for eight subjects with bilateral amygdala damage (gray squares) and 18 normal controls (bars, means $\pm S D$ ). Thus, correlations near 1 correspond to a normal rating profile given to that emotion, whereas low correlations correspond to an abnormal rating profile. Each emotion category consisted of six face stimuli depicting that emotion. While most autistic subjects performed normally, one subject gave a severely impaired performance when rating faces of fear, disgust, and surprise; and another subject gave an impaired performance when rating faces of disgust, a pattern of impairment similar to that seen in subjects with bilateral amygdala damage. Data for subjects with bilateral amygdala damage are from Adolphs et al. (1999), and details regarding the method of analysis can be found in Adolphs et al. (1995). 
7) gave very impaired performances when rating faces expressing fear, disgust, and surprise, a pattern of impairment that is similar to that seen in some subjects with bilateral amygdala damage. Another subject (Subject 2) also yielded abnormally low scores when rating disgust. The pattern of impaired recognition of fear, disgust, and surprise, with entirely normal recognition of happiness, is precisely what has been reported for subjects with bilateral amygdala damage (Adolphs, et al., 1999).

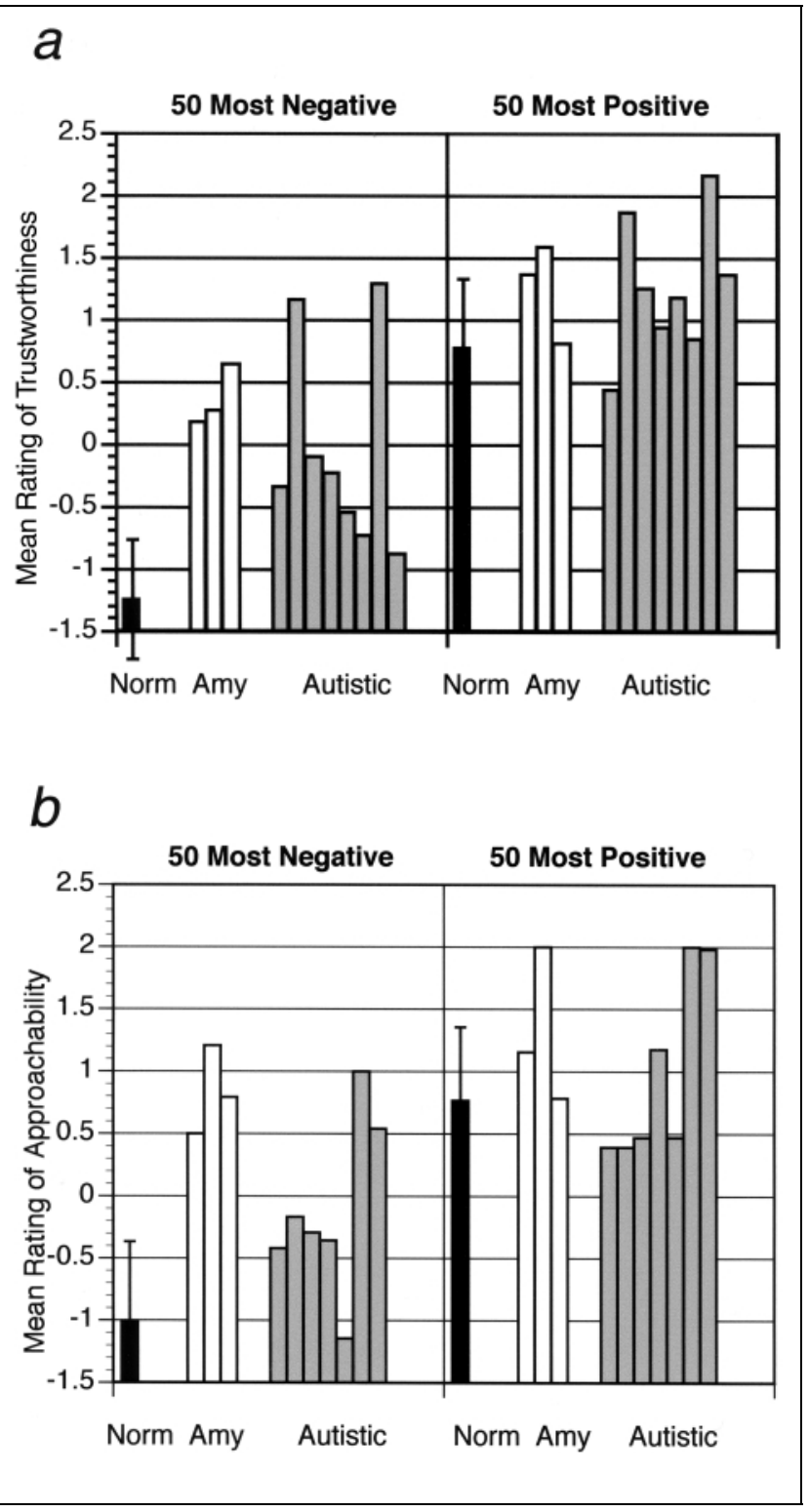

Figure 3. Ratings of (a) trustworthiness and (b) approachability from 100 unfamiliar faces. Shown are mean ratings of the 50 faces that normally receive the most negative ratings (left half of each split graph), and the 50 faces that normally receive the most positive ratings (right half of each split graph). Solid black bars are the means $( \pm S D)$ data from 47 normal controls; three white bars are data from three individual subjects with bilateral amygdala damage; gray bars are individual data from autistic subjects. All data except for the data from autistic subjects are from Adolphs et al. (1998). Norm = Normal; Amy = Amygdala .
Experiment 3. Social Judgment of Faces (Bellugi, Adolphs, Cassady, \& Chiles, 1999; Adolphs et al., 1998)

We analyzed data from these tasks separately in regard to those 50 stimuli that normally receive the most negative ratings, and those 50 stimuli that normally receive the most positive ratings. These are shown as the split graphs in Figure 3.

As a group, autistic subjects tended to give abnormally positive ratings to those 50 faces that normally receive the most negative ratings and there were some autistic subjects who gave highly abnormal ratings that were more positive than those given by any normal subject, as is also the case in subjects with bilateral amygdala damage. In particular, when rating that half of the stimuli that normally receive the most negative ratings with respect to trustworthiness (left half of Figure 3a), every autistic subject gave a mean rating that was more positive than the mean normal rating, and subjects 2 and 8 gave highly abnormally positive ratings of trustworthiness, a pattern previously reported for subjects with bilateral amygdala damage. Similarly, for ratings of approachability (Figure 3b), all but one of the autistic subjects gave ratings that were more positive than the mean control rating, and subjects 8 and 10 gave abnormally positive ratings to those half of the stimuli that normally receive the most negative ratings.

Despite the small sample sizes, some of these effects were statistically significant. When examining those 50 faces that normally receive the most negative trustworthiness ratings, Mann-Whitney $U$ tests revealed that both autistic subjects (corrected $p<.005$ ) and subjects with bilateral amygdala damage (corrected $p<.01$; all $p \mathrm{~s}$ are Bonferroni-corrected for multiple comparisons) gave higher ratings than did normal controls; but ratings given by autistic subjects did not differ significantly from those given by subjects with bilateral amygdala damage (corrected $p>$.8). For approachability ratings of the 50 faces that normally receive the most negative ratings, ratings given by autistic subjects did not differ significantly from either those given by subjects with bilateral amygdala damage (corrected $p>3$ ) or those given by normal controls (corrected $p>9$ ).

\section{Experiment 4. Social Judgment from Lexical Stimuli (Adolphs et al., 1998)}

When autistic subjects were given adjectives that describe personality attributes, they did not differ from normals in rating their likeability of those attributes (Mann-Whitney $U$ test comparing autistic subjects to normal controls; $p>$.6). In fact, ratings given by autistic subjects were highly correlated with ratings given by normal control subjects (Spearman rank correlations: Spearman $\rho=0.71-0.90$; all $p s<.0001)$. 
When given short biographies that described people, autistic subjects likewise gave normal ratings $(p>.8$; Mann-Whitney $U$ test) that correlated significantly with those given by normal controls (Spearman $\rho$ : 0.65-0.84; all $p s<.005)$. Both these above findings are very similar to those previously reported for subjects with bilateral amygdala damage, who also perform entirely normally in their social judgments of adjectives and biographies (Adolphs et al., 1998).

\section{DISCUSSION}

The findings of the present study can be summarized as follows. First, subjects with autism did not have any visuoperceptual impairments in discriminating human faces, either on the basis of identity or on the basis of emotional expression. These data make it unlikely that the impairments discussed below could be attributable to a simple perceptual impairment in processing the stimuli. In this regard, the findings from subjects with autism parallel those from subjects with focal damage to limbic structures important to social cognition, such as the amygdala and the orbitofrontal cortices: Such lesion patients also show a normal visuoperception of faces, despite impairments in linking visual percepts to the social and emotional information that such stimuli might signal (Adolphs et al., 1995; Damasio, 1994).

Second, while the findings on the other tasks show a large variance, there is an overall pattern, of which some, but not all, components bear similarity to what has been reported in subjects with bilateral amygdala damage. (a) Unlike subjects with bilateral amygdala damage, subjects with autism generally performed normally in their recognition of basic emotions from facial expressions. However, one of the subjects with autism showed impaired recognition of certain negative emotions from human facial expressions, notably fear, disgust, and surprise, a pattern of impaired emotion recognition that is also seen in some subjects with bilateral amygdala damage. (b) Like subjects with bilateral amygdala damage, autistic subjects gave ratings of trustworthiness that were more positive than the mean normal ratings, when judging those faces that normally receive the most negative ratings. Several autistic subjects gave highly abnormally positive ratings of trustworthiness and of approachability to unfamiliar faces. These findings are the most striking in our paper, as three out of the three subjects with bilateral amygdala damage gave abnormally positive ratings of trustworthiness, as did eight out of the eight subjects with autism. (c) Autistic subjects gave normal social judgments when the stimuli were lexical, as did subjects with bilateral amygdala damage.

While our results must be taken as preliminary, given the small sample sizes, they nonetheless suggest a pattern that is consistent with the hypothesis of amygdala dysfunction in a subset of subjects with autism. Taken together, the data suggest that some of the same processes that are impaired in subjects with bilateral amygdala damage may also be dysfunctional in some subjects with autism. The impairment we report appears to be most striking (and most similar to that reported in subjects with bilateral amygdala damage) for higher-level social judgments from faces (such as trustworthiness judgments), but not for recognizing basic emotions from faces.

It is worth reiterating that the performances given by subjects with autism were quite heterogeneous: Some subjects were impaired on some tasks, whereas other subjects were impaired on different tasks. An important future goal will be to investigate whether this heterogeneity reflects differences between subjects (e.g., some subjects had a particular brain pathology, and others not) or if it reflects heterogeneity in performances within a single subject. It is possible that autistic subjects, like subjects with bilateral amygdala damage (Adolphs et al., 1999) give variable performances, an issue that could be addressed with future retesting of the same subjects on the same tasks.

The overall pattern of results points towards a disproportionate impairment in those processes that subserve higher-level social cognition, with relative sparing of perceptual processing of faces, and of the recognition of basic emotions. The data are thus consistent with the idea that autistic subjects are able to form normal perceptual representations of faces (Experiment 1), and that they are able to retrieve knowledge regarding the basic emotion expressed (Experiment 2), but that they fail to link perception of the face to the social judgments called for in our task (Experiment 3)-possibly either because they cannot trigger normal retrieval of social knowledge, or because they have not acquired normal social knowledge to begin with. It is important to note that, like subjects with bilateral amygdala damage, the impaired social judgment disappears when the information is presented in a more explicit, lexical format (Experiment 4). This suggests that at least some basic social knowledge and some ability to form social judgments are intact in autism. It would appear that autism features dysfunction in those neural structures necessary to link percepts of visual, nonlexical stimuli with their social meaning, an interpretation we have previously put forth in regard to subjects with bilateral amygdala damage (Adolphs et al., 1995, 1998).

It is of interest also to compare the above findings with those from subjects with Williams Syndrome, a genetic disorder that, in some respects, presents the converse of the social impairments seen in autism (Bellugi, Lichtenberger, Mills, Galaburda, \& Korenberg, 1999). Subjects with Williams Syndrome are often hypersocial, and appear unusually empathic and skilled in their social interactions, in the face of severe impairments in other cognitive domains. We recently assessed social judgment in subjects with Williams Syndrome, using identical stimuli and a similar task to the one used 
here (Bellugi, Adolphs, et al., 1999). In that study, subjects with Williams Syndrome showed an abnormal positive bias in their social judgments, consistent with their real-life prosocial behavior. Surprisingly, the present report also found a positive bias in subjects with autism, although from their real-life behavior one might have expected a negative bias. While entirely speculative, one possibility would thus be that subjects with Williams Syndrome and subjects with autism both share some social processing impairments in common, and, moreover, that this impairment may result in part from amygdala dysfunction.

As we pointed out above, it is quite possible that our sample of autistic subjects may have been heterogeneous with respect to underlying brain abnormalities, so that only a subset of our subjects had amygdala dysfunction, and others had autistic behavior resulting from dysfunction in structures other than the amygdala. Furthermore, we wish to stress that, while the present findings may implicate the amygdala in some of the social dysfunction seen in autism, our data say nothing about the amygdala's role in accounting for other aspects of the autistic phenotype, nor do they imply that the amygdala is the only contributor. Indeed, it seems most probable to us that the amygdala will be only one component of a distributed neural system that is dysfunctional in autism (Damasio \& Maurer, 1978).

Comparisons between the present data and findings from other investigations of face processing in autism are made difficult by the differences in subject selection criteria, and differences in stimuli and procedures amongst studies. Furthermore, a limitation of the present study is the relatively small sample size. The literature on face processing in autism suggests a key abnormal component in regard to how individual features of faces are processed: While normal subjects tend to process faces holistically, there is some evidence that subjects with autism process them in a more analytical, feature-based fashion, and attend to different features than normals would (Hobson, Ouston, \& Lee, 1988b; Langdell, 1978). Autistic subjects were impaired in a task of facial emotion matching, when the faces to be matched were presented at separate times rather than concurrently, thus making feature-based strategies of comparison difficult, suggesting that holistic processing of facial emotion is abnormal in autism (Celani, Battacchi, \& Arcidiacono, 1999). Other studies have reported impairments in perceiving identity (Boucher, Lewis, \& Collis, 1998) or emotion (Capps, Yirmiya, \& Sigman, 1992; Hobson, Ouston, \& Lee, 1988a, Hobson, Ouston, \& Lee, 1989) from faces in autistic subjects, and a case study of Asperger Syndrome reported relatively impaired recognition of specific emotions (anger and disgust) in facial expressions (Ellis \& Leafhead, 1996). No study to date has carried out such investigations using the same experimental tasks that were used in the present paper.
Faces, as a close second possibly only to language, convey critical social information and are a key channel for social communication. The ability to use the face as a channel for social communication develops early in infancy, and was already noted as an apparent defect in subjects with autism by Kanner (1943). Despite the general consensus that autistic subjects do not process social and emotional information from faces normally, it has been difficult to ascertain precisely the nature of the impairment. The present study provides an additional piece of evidence, and suggests a possible set of specific processes that might be impaired in autism. Given the intact visuoperceptual discrimination of autistic subjects on our tasks, it appears plausible that early perceptual processing is intact, but that autism features an impaired ability subsequently to trigger normal retrieval of knowledge, and normal social behaviors, on the basis of the visual representations of faces. Possibly, amygdala dysfunction is one neuroanatomical correlate of this impaired mechanism, a hypothesis that will need to be tested in additional experiments with larger sample sizes, and that could be explored also with functional imaging studies of amygdala activation in autism.

\section{METHODS}

\section{Subjects}

Eight male subjects who all met DSM-IV/ICD-10 diagnostic criteria for autism participated in our studies. All subjects met the cutoff scores for autism on the Autism Diagnostic Interview (LeCouteur, Rutter, \& Lord, 1989), all had verbal and performance IQs in the normal range (from WAIS-R or WISC-R), and all had a normal ability to discriminate faces of different individuals, as determined by normal performances on the Benton Facial Recognition Task. Our sample, thus, comprised a group of highfunctioning autistic subjects (Table 1). All subjects were tested individually by the same experimenters in the same room.

\section{Experimental Tasks}

We used tasks that ranged from simple perceptual discrimination of emotions (Experiment 1), to recognition of the intensity of basic emotions in facial expressions (Experiment 2), to more subtle social judgments (Experiments 3 and 4). Tasks were administered in randomized order. There was no time limit for any task, and breaks were taken between and, in some cases, in the middle of tasks, as suited to particular subjects.

\section{Experiment 1: Discriminating the Intensity of Facial Emotion}

To provide a sensitive measure of the visuoperceptual ability with respect to the same class of stimuli (Ekman 
\& Friesen, 1976) used in some of the experimental tasks below (facial expressions of emotion), we administered a standardized two-alternative forced-choice discrimination task. Subjects were shown 80 pairs of faces. Each pair of faces was shown side-by-side, and the two faces in the pair differed in the intensity of emotion expressed. Subjects were asked to identify the face that showed the more intense emotion. The stimuli were computergenerated morphs that showed only a very faint emotion, permitting us to calculate a threshold above which subjects were able to discriminate the stimuli, and below which their performances were equivalent to chance. Six of the autistic subjects (Subjects 1, 4, 5, 6, 7, 8) participated in this task, and their data were compared to those from 28 normal subjects (Jansari et al., 2000) and from three subjects with bilateral amygdala damage (Adolphs et al., 1998).

\section{Experiment 2: Recognition of Basic Emotions}

This task has been used in several prior published reports to investigate recognition of the intensity of facial emotion following focal brain damage; (e.g., to demonstrate impaired recognition of emotion in subjects with bilateral amygdala damage; Adolphs et al., 1994, 1995, 1999). Briefly, subjects were shown 39 facial expressions of basic emotions (six instances each of happiness, surprise, fear, anger, disgust, and sadness, as well as three neutral faces) from Ekman and Friesen (1976) and asked to rate each face with respect to the intensity of each of the six basic emotions. Thus, a subject would see, e.g., a facial expression of fear, and be asked to rate that face with respect to the intensity of fear, happiness, surprise, anger, disgust, and sadness that they thought the depicted person would feel. Ratings from subjects were correlated with mean normal ratings to obtain a correlation score (Adolphs et al., 1994). Fisher $Z$-transforms of the correlations were used in all averaging procedures and in all parametric statistical analyses. Seven autistic subjects participated in this task (Subjects 1, 2, 4, 5, 7, 8, 10). Their scores were compared with an identical measure obtained from 18 normal control subjects, and from 8 subjects with amygdala damage (Adolphs et al., 1999).

\section{Experiment 3: Complex Social Judgments of Faces}

(a) Judgments of trustworthiness. Subjects were shown 100 faces of unfamiliar people and asked to judge how much they would trust the person, by giving a rating on a seven-point scale. The 100 stimuli had been carefully chosen so as to uniformly span the range of ratings from minimum to maximum, and so as to minimize the variance in the ratings given by normal subjects (cf. Adolphs et al., 1998). All eight autistic subjects participated in this task. Data were compared to those obtained from 47 normal controls, and to those from 3 subjects with bilateral amygdala damage (Adolphs et al., 1998).

(b) Judgments of approachability. Subjects were shown the same 100 faces as for the judgments of trustworthiness, and asked to judge how much they would like to approach the person and initiate a conversation with them if they were to meet them on the street. Seven of the autistic subjects (Subjects 1, 4, 5, 6, $7,8,10)$ participated in this task. Data were again compared to those obtained from 47 normal controls and 3 subjects with bilateral amygdala damage (Adolphs et al., 1998).

\section{Experiment 4: Complex Social Judgments of Lexical Stimuli}

(a) Words. We chose 88 adjectives that described personality attributes from a large standardized set (Anderson, 1968). We chose the words to span the range from very likeable to very dislikeable, and to exhibit maximal reliability and common usage (cf. Adolphs et al., 1998). Subjects were asked to rate on a seven-point scale how much they would like a person who exhibited the given adjective. Five of the subjects with autism (Subjects 1, 4, $5,6,7)$ participated in the task. Data were compared to those given by 20 normal controls, and could also be compared to those from three subjects with bilateral amygdala damage (Adolphs et al., 1998).

(b) Biographies. We constructed 20 short stories, which described people, containing information about the person's lifestyle and activities. Subjects were asked to rate how much they liked the individuals described by the stimuli, on a seven-point scale. Five autistic subjects participated in this task (Subjects 1, 4, 5, 7, 10). Data were compared to those given by 20 normal controls, and could also be compared to those from three subjects with bilateral amygdala damage (Adolphs et al., 1998).

\section{Note Added in Proof}

Since acceptance of our manuscript, several additional relevant studies have appeared. Critchley et al. (2000) found that high-functioning autistic subjects failed to show normal amygdala activation in response to implicit processing of emotional facial expressions. In a study that bears some similarity to ours, Howard et al. (2000) found impaired recognition of emotion from facial expressions, as well as abnormalities on structural MR images of the amygdala, in subjects with autism. A study by Grossman, Klin, Carter and Volkmar (2000) found, as we did, that high-functioning subjects with autism may not be impaired in simple recognition of basic emotions from faces, but that they are impaired on more demanding social judgments from faces; the authors proposed that autism may feature impaired emotion recognition, but that high-functioning subjects may be able to com- 
pensate in part for their impairment via strategies that use verbal mediation. Taken together, these recent reports corroborate our present findings that autism may result from an impaired ability to link perception of faces to the retrieval of social knowledge, and that this impairment may result, in part, from dysfunction involving the amygdala.

\section{Acknowledgments}

We thank Jeremy Nath and Ashok Jansari for help in testing subjects. Supported in part by grants from NIMH, the Sloan Foundation, and the EJLB Foundation to R.A.

Reprint requests should be sent to: Ralph Adolphs, Department of Neurology, University of Iowa Hospitals and Clinics, 200 Hawkins Drive, Iowa City, IA 52242, USA. E-mail: ralphadolphs@uiowa.edu.

\section{REFERENCES}

Abell, F., Krams, M., Ashburner, J., Passingham, R., Friston, K., Frackowiak, R., Happe, F., Frith, C., \& Frith, U. (1999). The neuroanatomy of autism: A voxel-based whole brain analysis of structural scans. NeuroReport, 10, 1647-1651.

Adolphs, R. (1999). Social cognition and the human brain. Trends in Cognitive Sciences, 3, 469-479.

Adolphs, R., Tranel, D., \& Damasio, A. R. (1998). The Human amygdala in social judgment. Nature, 393, 470-474.

Adolphs, R., Tranel, D., Damasio, H., \& Damasio, A. (1994). Impaired recognition of emotion in facial expressions fol lowing bilateral damage to the human amygdala. Nature, 372, 669-672.

Adolphs, R., Tranel, D., Damasio, H., \& Damasio, A. R. (1995). Fear and the human amygdala. The Journal of Neuroscience, 15, 5879-5892.

Adolphs, R., Tranel, D., Hamann, S., Young, A., Calder, A., Anderson, A., Phelps, E., \& Damasio, A. R. (1999). Recognition of facial emotion in nine subjects with bilateral amygdala damage. Neuropsychologia, 37, 1111-1117.

Anderson, N. H. (1968). Likableness ratings of 555 personalitytrait words. Journal of Personality and Social Psychology, 9, 272-279.

Bachevalier, J. (1991). An animal model for childhood autism: Memory loss and socioemotional disturbances following neonatal damage to the limbic system in monkeys. In C. A. Tamminga \& S. C. Schulz (Eds.), Advances in neuropsychiatry and psychopharmacology, vol. 1, Schizophrenia research (pp. 129-140). New York: Raven.

Baron-Cohen, S. (1995). Mindblindness: An essay on autism and theory of mind. Cambridge: MIT Press.

Baron-Cohen, S., Ring, H. A., Bullmore, E. T., Wheelwright, S., Ashwin, C., \& Williams, S. C. R. (2000). The amygdala theory of autism. Neuroscience and Biobehavioral Reviews, 24, 355-364.

Baron-Cohen, S., Ring, H. A., Wheelwright, S., Bullmore, E. T., Brammer, M. J., Simmons, A., \& Williams, S. C. R. (1999). Social intelligence in the normal and autistic brain: An fMRI study. European Journal of Neuroscience, 11, 1891-1898.

Baron-Cohen, S., Spitz, A., \& Cross, P. (1993). Can children with autism recognize surprise? Cognition and Emotion, 7, 507-516.

Baron-Cohen, S., Wheelwright, S., \& Jolliffe, T. (1997). Is there are a "language of the eyes"? Evidence from normal adults and adults with autism or Asperger Syndrome. Visual Cognition, 4, 311-332.
Bauman, M., \& Kemper, T. L. (1985). Histoanatomic observations of the brain in early infantile autism. Neurology, 35 , 866-874.

Bellugi, U., Adolphs, R., Cassady, C., \& Chiles, M. (1999). Towards the neural basis for hypersociability in a genetic syndrome. NeuroReport, 10, 1653-1659.

Bellugi, U., Lichtenberger, L., Mills, D., Galaburda, A., \& Korenberg, J. R. (1999). Bridging cognition, the brain, and molecular genetics: Evidence from Williams syndrome. Trends in Neurosciences, 22, 197-207.

Benton, A. L., Hamsher, K., Varney, N. R., \& Spreen, O. (1983). Contributions to neuropsychological assessment. New York: Oxford University Press.

Boucher, J., Lewis, V., \& Collis, G. (1998). Familiar face and voice matching and recognition in children with autism. Journal of Child Psychology and Psychiatry, 39, 171-181.

Breiter, H. C., Etcoff, N. L., Whalen, P. J., Kennedy, W. A., Rauch, S. L., Buckner, R. L., Strauss, M. M., Hyman, S. E., \& Rosen, B. R. (1996). Response and habituation of the human amygdala during visual processing of facial expression. Neuron, 17, 875-887.

Broks, P., Young, A. W., Maratos, E. J., Coffey, P. J., Calder, A. J., Isaac, C., Mayes, A. R., Hodges, J. R., Montaldi, D., Cezayirli, E., Roberts, N., \& Hadley, D. (1998). Face processing impairments after encephalitis: Amygdala damage and recognition of fear. Neuropsychologia, 36, 59-70.

Capps, L., Yirmiya, N., \& Sigman, M. (1992). Understanding of simple and complex emotions in non-retarded children with autism. Journal of Child Psychology and Psychiatry, 33, 1169-1182.

Celani, G., Battacchi, M. W., \& Arcidiacono, L. (1999). The understanding of the emotional meaning of facial expressions in people with autism. Journal of Autism and Developmental Disorders, 29, 57-66.

Critchley, H. D., Daly, E. M., Bullmore, E. T., Williams, S. C., van Amelsvoort, T., Robertson, D. M., Rowe, A. M. P., McAlonan, G., Howlin P., \& Murphy, D. G. (2000). The functional neuroanatomy of social behavior: Changes in cerebral blood flow when people with autistic disorder process facial expressions. Brain, 123, 2203-2212.

Damasio, A. R. (1994). Descartes' error: Emotion, reason, and the buman brain. New York: Grosset/Putnam.

Damasio, A. R., \& Maurer, R. G. (1978). A neurological model for childhood autism. Archives of Neurology, 35, 777-786.

Ekman, P., \& Friesen, W. (1976). Pictures of facial affect. Palo Alto, CA: Consulting Psychologists Press.

Ellis, H. D., \& Leafhead, K. M. (1996). Raymond: A study of an adult with Asperger syndrome. In P. W. Halligan \& J. C. Marshall (Eds.), Methods in madness: Case studies in cognitive neuropsychiatry (pp. 79-92). Hove, UK: Psychology Press.

Grossman, J. B., Klin, A., Carter, A. S., \& Volkmar, F. R. (2000). Verbal bias in recognition of facial emotions in children with Asperger syndrome. Journal of Child Psychology and Psychiatry, 41, 369-379.

Hobson, R. P., Ouston, J., \& Lee, A. (1988a). Emotion recognition in autism: Co-ordinating faces and voices. Psychological Medicine, 18, 911-923.

Hobson, R. P., Ouston, J., \& Lee, A. (1988b). What's in a face? The case of autism. British Journal of Psychology, 79, 441453.

Hobson, R. P., Ouston, J., \& Lee, A. (1989). Naming emotion in faces and voices: Abilities and disabilities in autism and mental retardation. British Journal of Developmental Psychology, 7, 237-250.

Howard, M. A., Cowell, P. E., Boucher, J., Broks, P., Mayes, A., Farrant, A., \& Roberts, N. (2000). Covergent neuroanatomical and behavior evidence of an amygdala hypothesis of autism. NeuroReport, 11, 2931-2935. 
Jansari, A., Tranel, D., \& Adolphs, R. (2000). A valence-specific lateral bias for discriminating emotional facial expressions in free-field. Cognition and Emotion (in press).

Kanner, L. (1943). Autistic disturbances of affective contact. Nervous Child, 2, 217-250.

Karmiloff-Smith, A., Bellugi, U., Klima, E., Grant, J., \& BaronCohen, S. (1995). Is there a social module? Language, face processing, and theory of mind in individuals with Williams Syndrome. Journal of Cognitive Neuroscience, 7, 196-208.

Langdell, T. (1978). Recognition of faces: An approach to the study of autism. Journal of Child Psychology and Psychiatry, 19, 255-268.
LeCouteur, A., Rutter, M., \& Lord, C. (1989). Autism diagnostic interview: A standardized investigator-based instrument. Journal of Autism and Developmental Disorders, 19, 363387.

Leslie, A. (1987). Pretense and representation: The origins of "theory of mind". Psychological Review, 94, 412-426.

Morris, J. S., Frith, C. D., Perrett, D. I., Rowland, D., Young, A. W., Calder, A. J., \& Dolan, R. J. (1996). A differential neural response in the human amygdala to fearful and happy facial expressions. Nature, 383, 812-815.

Piven, J. (1997). The biological basis of autism. Current Opinion in Neurobiology, 7, 708-712. 